Western North American Naturalist 67(4), (C) 2007, pp. 520-523

\title{
MOISTENED SEEDS INCREASE RODENT TRAP SUCCESS
}

\author{
John C. Tull1,3 and Michael W. Sears ${ }^{2,4}$
}

\begin{abstract}
Seed moisture has been shown to influence the rates of seed cache removal by rodents. Although the precise mechanism is not known, this knowledge might prove useful in field applications. We examined whether moistened bait would increase trap success in desert rodent populations. We placed traps $15 \mathrm{~m}$ apart in grids within a 500-ha study area and randomly baited traps with either dry or moistened seeds. We found that traps baited with moistened seeds had $34.9 \%$ higher success than traps baited with dry seeds $\left(n=190, \chi^{2}=5.389\right.$, $\left.\mathrm{df}=1, P=0.020\right)$. Our results suggest that application of water to dry seed bait can lead to increased trap success for desert rodents.
\end{abstract}

Key words: seed moisture, trap success, Heteromyidae, bait, live-trap, rodent, small mammal.

Trap success can influence the efficiency and precision of mark-recapture studies. Species accumulation curves are typically used to determine when trapping effort can be declared adequate because they reveal when new individuals or species are no longer being added with additional trap effort (Jones et al. 1996). With low trap success, studies on species diversity may fail to adequately represent the richness of species in a sampled area because of the constraints of time, available personnel, equipment, and money. Estimates of abundance similarly suffer from reduced precision per unit effort with lower trap success. Improving trap success should therefore improve the overall efficiency of such studies by reducing the amount of time required to trap at a site. For studies that estimate abundance, if more individuals in the sample population are being trapped per unit effort, then the overall abundance estimates may be more accurate.

One way to improve capture probabilities is to lure animals to traps by using baits that are highly attractive (Jones et al. 1996). In terms of dietary requirements, desert heteromyids may survive by using metabolic water when surface moisture is unavailable (MacMillen and Christopher 1975, MacMillen and Hinds 1983). Also, the moisture content of food items may be an important factor in foraging decisions of desert rodents (Murray and Dickman 1994).

Heteromyid rodents are better able to find cached seeds with high moisture content rel- ative to seeds with low moisture content (Vander Wall 1993), and they also find seeds more readily in moist versus dry substrates (Vander Wall 1995, Vander Wall et al. 2003). Greater cache recovery of moist seeds is likely due to a triggered release of odorant molecules from the seed under moist conditions (Vander Wall 1998, 2003). Regardless of the exact mechanisms, it is clear that moisture promotes detectability of seeds for at least some granivorous rodents.

The apparent improved detection of moist seeds by desert rodents may have practical field application for ecological studies reliant on animal captures. Specifically, moistened seed bait may produce higher trap efficiency than standard dry seed bait, especially during hot and dry months, when ecological studies on desert rodents often occur and when environmental moisture is quite low. Our objective was to test the effect of seed moisture on trap success of unique animals (i.e., animals not previously trapped). We hypothesized that heteromyid rodents would be captured more often in traps baited with moistened seeds versus dry seeds.

\section{Methods}

Our study area was located in Great Basin desert scrub habitat, $\sim 3 \mathrm{~km}$ north of Soda Lake, Fallon, Churchill County, Nevada. Research occurred predominantly within stabilized sand dunes over a $<500$-ha area. Vegetation was dominated by saltbush (Atriplex spp.) and horsebrush (Tetradymia spp.).

\footnotetext{
${ }^{1}$ Program in Ecology, Evolution and Conservation Biology, University of Nevada, Reno, NV 89557.

2Department of Biology, University of Nevada, Reno, NV 89557.

3Present address: Department of Biology, Mailstop 314, University of Nevada, Reno, NV 89557. E-mail: jctull@biodiversity.unr.edu

${ }^{4}$ Present address: Department of Zoology, Southern Illinois University, Carbondale, IL 62901.
} 
We trapped rodents in August 2002 and June 2003 using aluminum and galvanized folding Sherman live-traps that were $7.6 \mathrm{~cm}$ wide, $8.9 \mathrm{~cm}$ tall, and $22.9 \mathrm{~cm}$ long (H.B. Sherman Traps, Tallahassee, FL). All trapping occurred during dry weather with low relative humidity, and on days when precipitation had not occurred for at least 5 days. We placed trap grids in either a $10 \times 10$ configuation $(100$ traps) or a $10 \times 12$ configuration (120 traps) with $15 \mathrm{~m}$ between rows and columns of the grid. We placed grids in nonoverlapping geographic space, and each grid was only trapped for a single night to reduce animal recaptures and to maintain independence for our experimental unit, the trap. Between trap sessions we placed individual traps indiscriminately in relation to grid location.

We laid the traps prior to baiting them to minimize dessication of the moistened seed bait. We baited traps no sooner than 30 minutes before sunset and completed baiting $\leq 45$ minutes after sunset. We specifically designed our experiment to treat the trap as the experimental unit so that we would be able to generalize our result to individual trap success. Treatments (moistened seeds or dry seeds) were randomly assigned to individual traps in equal numbers per treatment group for each trapping grid. Consequently, any trap preferences that may have existed were randomized and would be expressed as random error in the statistical analysis.

We moistened seeds by mixing $120 \mathrm{~mL}$ of deionized water with $1000 \mathrm{~g}$ of wild bird seed (millet, milo, and sunflower blend) in a ziplock bag 10 minutes prior to the onset of trap baiting. Dry seed bait was stock seed stored in the shade without additional moisture added. Dry seed bait and moistened seed bait were handled separately to avoid cross-contamination of odor and moisture from 1 source to the other. We kept the moistened seeds out of sun exposure to avoid desiccation of the treated bait during handling. We added approximately 35 $\mathrm{mL}$ by volume of either moistened or dry seeds to each trap with separate measuring spoons for each bait type. We never made direct contact with either seed type to minimize the possible effects of human scent on bait handling (Duncan et al. 2002, Wenny 2002). We did not attempt to measure seed moisture content between moistened and dry seed bait because we were only interested in how the addition of moisture to seed bait affected trap success.

We left traps open for $\sim 12$ hours overnight and checked traps for rodents the following morning. We marked newly captured animals for each trap period with an ear punch. We recorded species, bait type (i.e., moistened vs. dry), and trap location, and then released the animal at the trap site. We collected traps each morning, and there was no residual moisture in traps when we placed them out again. Although we made no specific attempt to remove odors from traps between trapping episodes, our randomization of treatment groups to traps would treat any influence of residual odors as random error in the analysis.

We performed the $\chi^{2}$ goodness-of-fit test to examine the treatment effect (Zar 1984) excluding both non-heteromyid rodents and recaptured animals that moved between trap grids. Our analyses were performed in program Octave (Eaton 2002).

\section{REsults}

We had 820 trap-nights of effort over 7 nights with equal numbers of traps baited with either moistened or dry seeds. Overall we captured 215 animals, of which 20 were recaptures, for a total trap success of $23.8 \%$ for unique animals. Of the 195 initial captures, most were heteromyid rodents (97.4\%), including 118 (60.5\%) Merriam's kangaroo rats (Dipodomys merriami), 55 (28.2\%) little pocket mice (Perognathus longimembris), 12 (6.2\%) pallid kangaroo mice (Microdipodops pallidus), and $5(2.6 \%)$ Ord's kangaroo rats $(D$. ordii); we also caught $1(0.5 \%)$ white-tailed antelope squirrel (Ammospermophilus leucurus) and $4(2.0 \%)$ grasshopper mice (Onychomys spp.; Fig. 1).

We found a $34.9 \%$ increase in trap success through the use of moistened seeds relative to dry seed bait. The total number of initial captures in each treatment was 111 for moistened seed bait and 79 for dry seed bait (Fig. 1). Heteromyid rodents were trapped more frequently in traps baited with moist seeds compared to dry seeds $\left(\chi^{2}=5.389, \mathrm{df}=1, P=0.020\right)$.

\section{Discussion}

Our experiment showed that adding moisture to seed bait can significantly improve trap success for heteromyid rodents. Our result 


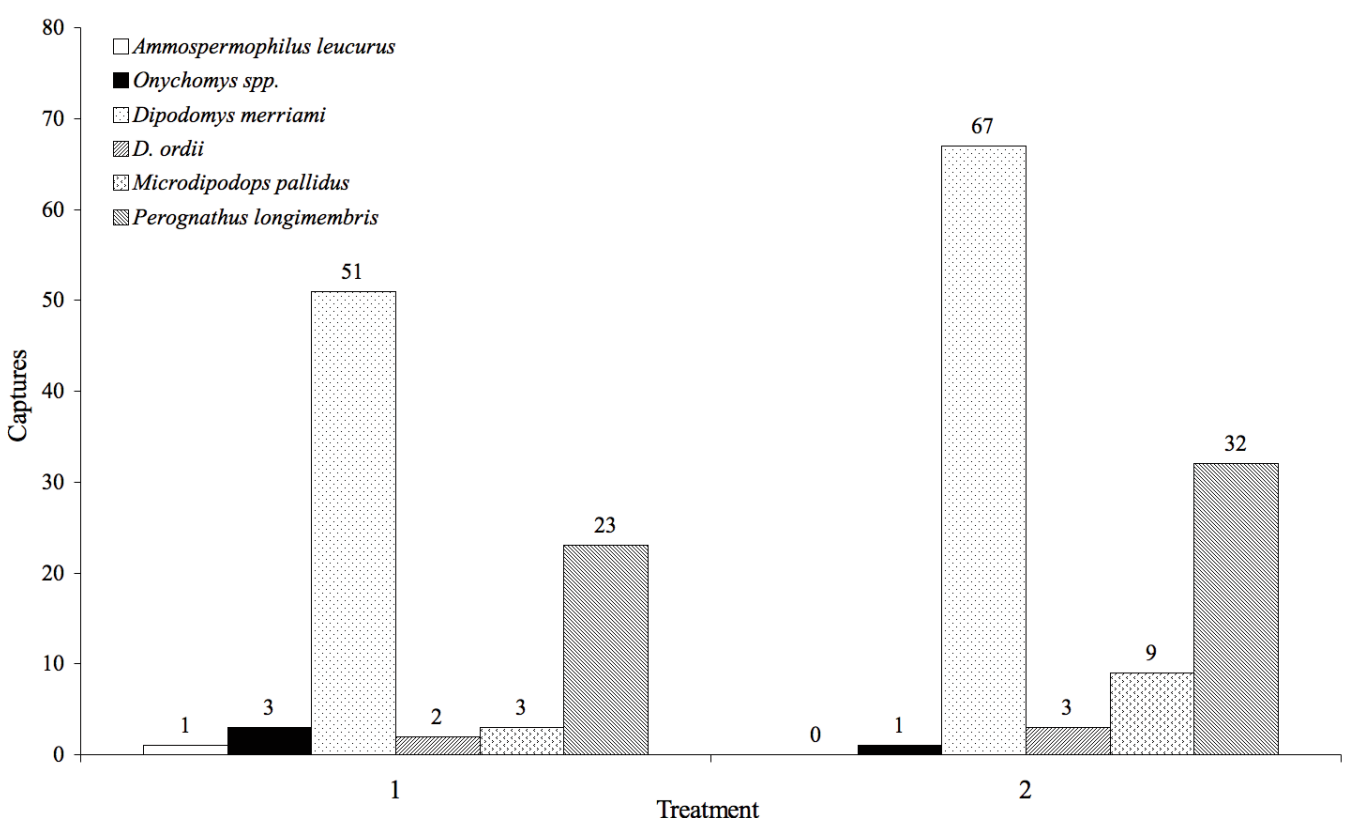

Fig. 1. First-time captures for rodents in each treatment, dry seed bait (treatment 1 ) and moistened seed bait (treatment 2), over 820 trap-nights of effort in Churchill County, Nevada, during August 2002 and June 2003.

provides additional evidence that granivorous rodents are capable of discriminating seeds with high moisture content (Vander Wall 1993, Murray and Dickman 1997), and that heteromyids will select moistened seeds over dry seeds when both are available.

Although it is known that heteromyid rodents find cached seeds in moist environments at higher rates than in dry substrates (Vander Wall 1995) and at higher rates when the seeds themselves are moist (Vander Wall 1993), the use of moistened seeds has not been previously tested as a means of providing a straightforward and inexpensive technique for improving trap success for heteromyid rodents. Although the theoretical basis is not in place to support the idea that moist seed baits will improve trap success in all ecological conditions, it would be interesting to explore this further in a mesic environment and in an area with greater rodent diversity (e.g., in a study not primarily limited to heteromyid rodents). Such studies would be able to address the hypothesis that granivorous rodents are more capable than other rodents of finding seeds with high moisture content (Murray and Dickman 1997). Heteromyid rodents dominated our study site, so a comparison between desert-adapted species and other granivorous species simply was not possible.

We found a $34.9 \%$ increase in trap success through the use of moistened seeds relative to dry seed bait for heteromyids. The application of water to seed bait in relatively small quantities provides a simple yet effective means of improving capture success in field studies of desert rodents. Because we designed our study to treat the trap as the experimental unit, our finding that trap-specific capture probability is greater for traps baited with moistened seed bait should scale up to the typical unit of measure in small-mammal trapping studies, namely, the grid, transect, or web. Our technique is convenient and inexpensive to apply to standard mark-recapture studies or to other studies employing the use of baited traps.

\section{ACKNOWLEDGMENTS}

We wish to thank S. Vander Wall for generating our interest in this experiment and for reviewing a draft of this manuscript. J.C. Tull received financial support from the Whittell Forest and Wildlife Area Summer Research Fellowship program and from the Nevada Biodiversity Initiative. Our manuscript was improved by comments from 2 anonymous reviewers. 
Sherman traps were provided by the Biology Department at the University of Nevada, Reno. Trapping was performed under Nevada Division of Wildlife Permit \#S22548. The University of Nevada, Reno, Institutional Animal Care and Use Committee approved all trapping protocols (\#A01/02-36 Version 1).

\section{Literature Cited}

Duncan, R.S., D.G. Wenny, M.D. SpRITZer, and C.J. WheLan. 2002. Does human scent bias seed removal studies? Ecology 83:2630-2636.

Eaton, J.W. 2002. GNU octave manual. 3rd edition. Network Theory Ltd., Bristol, U.K.

Jones, C., W.J. McShea, M.J. Conroy, and T.H. Kunz. 1996. Capturing mammals. Pages 115-155 in D.E. Wilson, F.R. Cole, J.D. Nichols, R. Rudran, and M.S Foster, editors, Measuring and monitoring biological diversity: standard methods for mammals. Smithsonian Institution Press, Washington, DC.

MacMillen, R.E., and E.A. Christopher. 1975. The water relations of two populations of noncaptive desert rodents. Pages 117-137 in N.F. Hadley, editor, Environmental physiology of desert organisms. Dawden Hutchison \& Ross, Stroudsberg, PA.

MaCMillen, R.E., AND D.S. Hinds. 1983. Water regulatory efficiency in heteromyid rodents: a model and its application. Ecology 64:152-164.
Murray, B.R., and C.R. Dickman. 1994. Food preferences and seed selection in 2 species of Australian desert rodents. Wildlife Research 21:647-655.

1997. Factors affecting selection of native seeds in two species of Australian desert rodents. Journal of Arid Environments 35:517-525.

Vander WaLL, S.B. 1993. Seed water content and the vulnerability of buried seeds to foraging rodents. American Midland Naturalist 129:272-281.

. 1995. Influence of substrate water on the ability of rodents to find buried seeds. Journal of Mammalogy 76:851-856.

. 1998. Foraging success of granivorous rodents: Effects of variation in seed and soil water on olfaction. Ecology 79:233-241.

2003. How rodents smell seeds: a model based on the behavior of pesticides in soil. Journal of Mammalogy 84:1089-1099.

VAnder Wall, S.B., M.J. Beck, J.S. BRiggs, J.K. Roth, T.C. Thayer, J.L. Hollander, and J.M. Armstrong. 2003. Interspecific variation in the olfactory abilities of granivorous rodents. Journal of Mammalogy 84:487496.

WENnY, D.G. 2002. Effects of human handling of seeds on seed removal by rodents. American Midland Naturalist 147:404-408.

ZAR, J.H. 1984. Biostatistical analysis. 2nd edition. PrenticeHall, Inc., Englewood Cliffs, NJ.

Received 7 December 2006 Accepted 16 April 2007 